\title{
Education
}

\section{The travelling athlete: issues relating to the Commonwealth Games, Malaysia, 1998*}

\author{
Mark Young, Peter Fricker, Ron Maughan, Domhnall MacAuley
}

Australian Institute of Sport, Leverrier Crescent, Bruce ACT 2617, Belconnen ACT 2616, Australia $M$ Young

P Fricker

Department of Environmental and Occupational Medicine, University Medical School, Foresterhill, Aberdeen AB9 2ZD, UK

R Maughan

Hillhead Family Practice, Belfast BT11 9FZ, N Ireland, UK D MacAuley

Correspondence to: $\mathrm{M}$ Young.

Accepted for publication 11 December 1997

* This article will also appear in Clin $\mathcal{F}$ Sports Med 1998; April.
The international athlete is a global traveller. Such is the ease and speed of air travel that major international sporting events now take place throughout the world. This presents a variety of problems for the medical team and includes issues such as immunisation, vaccinations, jet lag, diet, and acclimatisation. The key to team care is preparation and, with the 1998 Commonwealth Games due to take place in Kuala Lumpur, Malaysia, in September, preparation must begin early. The main issues for the team of medical care providers relate to the three important phases in medical care: concerns before travel, minimising problems during travel, and providing care at the competition.

\section{Before travel}

The medical team should begin planning early in the preparation phase focusing on issues such as general health screening, vaccination and immunisation, and preparation for a change in climate. The medical officer in charge should coordinate all aspects of medical and dental screening and provide direction on appropriate intervention (table 1). The aim of early intervention is the prevention of problems that could affect training and preparation before travel, and to minimise the need for medical or dental care at the time of competition. Medical screening includes a general medical examination, but should focus in particular on identifying those conditions that can be treated preseason and thus prevent later problems during competition. Such conditions include inguinal hernia, impacted wisdom teeth, asthma, skin infections, anaemia, and iron deficiency for example. This consultation also offers an opportunity to advise on general medical issues and in particular on the hazards of overtraining and the importance of an appropriate diet and rest. Those athletes taking medications that require prior notification to drug testing authorities should be identified

Table 1 Key issues for the travelling athlete

The travelling athlete:
is susceptible to respiratory tract infection
may travel at short notice and require immediate prophylaxis
requires long term prophylaxis
needs to remain healthy to perform well
may not take responsibility for medical needs
exercises in different environments

The travelling athlete:

is susceptible to respiratory tract infection may travel at short notice and require immediate prophylaxis needs to remain healthy to perform well exercises in different environments and the notification provided. The location of the Games will expose competitors to the additional hazards of heat and humidity, so it is important to identify any competitors who have had previous problems with heat adaptation, heat stroke, or dehydration. Team members selected relatively late pose particular problems and some contingency should be included in planning.

This paper addresses the needs of the athlete, but it is pertinent to consider the needs of coaches and officials who accompany athletes, as they too may seek advice from the medical team. Often their medical requirements extend to conditions such as hypertension, diabetes mellitus, and coronary heart disease, and they should be included in any medical briefings.

IMMUNISATION, VACCINATION, AND PROPHYLAXIS When travelling to a developing country, there are usually specific health care recommendations. Table 2 summarises the recommended immunisation and prophylactic measures for the travelling athlete. These include general measures as well as those specific to competing in Malaysia. Some athletes may be at more risk than others depending on the nature of the sport, location of a pre-event training camp, and travel plans after the Games. For example, malaria prophylaxis is strongly advised for those training or residing out of Kuala Lumpur. Those athletes confident of selection should undertake appropriate immunisation procedures well before departure, and team selection should allow sufficient time for adequate immunisation/vaccination well before the Games. Late selection may make this difficult.

We include general recommendations, but travel advice can change, and we recommend that athletes and officials seek specialist advice on immunisation and prophylaxis closer to the date of departure. Appropriate advice can be obtained from local and national travel advisory services and from databases such as the internet web sites of the World Health Organisation and the Center for Disease Control, Atlanta.

All athletes should have had the basic childhood immunisations including diphtheria, tetanus, pertussis, and polio, and, if necessary, be vaccinated against measles, mumps, and 
Table 2 Immunisation for the athlete

\begin{tabular}{l}
\hline Basic/essential \\
Tetanus \\
Diphtheria \\
Measles \\
Mumps \\
Poliomyelitis \\
Rubella \\
Recommended \\
Hepatitis A (frequent \\
international travel) \\
Hepatitis B (contact sports \\
especially) \\
Influenza \\
Regional/geographic \\
Malaria \\
Typhoid fever \\
Japanese encephalitis \\
Cholera \\
Rabies \\
Meningococcus \\
Yellow fever \\
\hline
\end{tabular}

rubella. They should have had booster doses of oral polio vaccine and adsorbed diphtheria and tetanus vaccine within the previous ten years. Because of potentially higher risk, athletes playing on outdoor surfaces (especially soil) should receive five yearly adsorbed diphtheria and tetanus boosters. (Some caution should be exercised as boosters given too frequently can produce a severe local reaction at the injection site, the "hyperimmune" response.)

All travelling athletes should be immunised against hepatitis A. The optimal regime is an intramuscular dose of inactivated hepatitis A vaccine (1440 enzyme linked immunosorbent assay (ELISA) units) which is repeated at six to twelve months. This provides excellent short and long term protection against one of the commonest serious preventable infections acquired by international travellers.

Similarly, hepatitis B vaccination is recommended, especially for those who participate in contact sports such as boxing, wrestling, and football. The standard regime can be "accelerated" by giving three intramuscular injections of $20 \mu \mathrm{g}$ hepatitis B vaccine over three weeks, followed by a booster injection (same dose) at 12 months. This has several advantages over the six month protocol in that early seroprotection is achieved at one month ( $68 \%$ compared with $15.2 \%$ for the six month protocol) and late seroprotection is also improved $(100 \%$ compared with $97.4 \%) .{ }^{1}$ With the accelerated regime there is no need for follow up blood tests to assess immunity, and the initial and final injections can be given together with hepatitis A vaccinations.

Travelling athletes are prone to common viral infections including influenza because of air travel, cramped living conditions, and high training loads. Athletes should therefore have the influenza vaccine. It provides good immunity to three strains of virus and has few side effects.

Travelling to Malaysia may put athletes at particular risk so they should consider the following additional measures.

Antimalarial chemoprophylaxis may be required for those training and residing outside of Kuala Lumpur. Although malaria is uncommon on the Malay peninsula, chloroquine resistant falciparum malaria is present. Guidelines may vary, but in the United Kingdom mefloquine (Lariam) is recommended as the drug of choice. There has been recent adverse publicity about mefloquine, and the athlete should be counselled appropriately. The side effects of mefloquine include fine motor incoordination, vivid nightmares, and other neuropsychiatric effects. ${ }^{2}$ In Australia the recommended prophylaxis is doxycycline 100 mg daily for two days before, during, and for two to four weeks after leaving the malarial area. This regime is not licensed in the United Kingdom. Side effects of doxycycline include photosensitivity and thrush. For those with such sensitivity, chloroquine with proguanil, or mefloquine can be used.

As typhoid fever also occurs in Malaysia, prophylaxis is recommended for those attending the Commonwealth Games. At present the preferred vaccines are salmonella typhi Vi vaccine (Typhim Vi) or an oral live attenuated vaccine (Typh-vax). The former is a single intramuscular injection which provides good immunity for three years, and the latter gives five years of protection after four capsules are taken (as one capsule every second day).

Rabies prophylaxis can be given as the human diploid cell vaccine (three $0.1 \mathrm{ml}$ intradermal injections over three weeks). This is cost effective because the vaccine is provided in $1.0 \mathrm{ml}$ vials, and the regime is ideal for simultaneous vaccinations of groups of athletes. Post-exposure human rabies immunoglobulin should be given if an athlete is bitten by a wild animal (such as a monkey or wild dog, which are common in this area).

Japanese encephalitis and cholera may pose a risk to athletes at more remote parts of Malaysia. Appropriate immunisation is therefore recommended.

Malaysian customs and immigration officials may request a doctor's letter stating the purpose of any medication in the athlete's possession (including antimalarial drugs). They can also demand that anyone that they consider likely to transmit HIV leave the country immediately.

\section{Travel phase}

The circadian rhythms (related to the daynight cycle) may take seven days to normalise after more than five time zones have been crossed. ${ }^{3}$ The period of adjustment is up to $50 \%$ longer with eastbound travel because most circadian rhythms are slightly longer than 24 hours, and shortening a day produces more physiological stress. Adjustment also takes longer with increasing age, the mental stress associated with travel, and alcohol consumption.

"Jet lag" refers to the effects of rapid transmeridian travel and includes bowel disturbance, fatigue, and poor sleep. It is difficult to distinguish from "jet stress" which is attributed to the dehydrating effect of air travel, pressurised cabins, diet, lack of space, and noise.

Most studies measuring the effect of jet lag on athletic performance have methodological problems. ${ }^{4}$ However, it is prudent to give advice on minimisation of jet lag. If it is possible, athletes can prepare for the new time zone (of competition) by training, eating, and sleeping at the same times as they would be at the competition venue. This means deliberately shifting their daily routine by the appropriate number of hours necessary to facilitate this. After arrival, one should allow one day for each time zone difference to adjust to the new time zone. With a three hour time difference, few symptoms are experienced, but for greater time differences, preparation and adjustment are required. For Kuala Lumpur, athletes from different Commonwealth countries will have different requirements.

To minimise problems, athletes are encouraged to sleep well the night before departure, to synchronise their watches with the current time at destination on boarding, and to minimise the time spent in transit lounges by appropriate 
flight scheduling. It is important to plan specific individualised sleeping strategies. Some athletes prefer to "cat nap" throughout the flight, whereas others prefer to establish new sleep-wake cycles by planned sleeping times. Relaxation techniques, eye shields, ear plugs, and short acting hypnotics or melatonin may be helpful. Melatonin is a peptide secreted by the pineal gland especially at the onset of sleep. Exogenous melatonin, when given during and after air travel (over several days), has been shown to reduce subjective feelings of jet lag. ${ }^{5}$ Ideally the dose of hypnotic or melatonin should be given at bed time at the venue. A dose of $5 \mathrm{mg}$ melatonin is suggested ${ }^{6}$ but this drug, although available in variable doses in the US, is not widely available in Europe or Australia. It would be foolish to use either of the above for the first time when travelling to a major event such as the Commonwealth Games, and athletes should have experimented beforehand on a less important occasion. The British Olympic Association does not, however, recommend the use of melatonin.

To minimise the stress of jet travel, athletes should arrive early at the airport, arrange appropriate in-flight meals, wear loose comfortable clothing, and carry personal material for relaxation (such as books, magazines, and music). Athletes must always be prepared for delays and stressful events such as lost luggage. Bulkhead or aisle seats should be booked for tall athletes and all athletes should stretch and move around the cabin from time to time during the flight. There is limited food and drink available during a flight and the airline is accustomed to catering for the average passenger rather than a hungry athlete. The athlete should therefore carry extra food and drinks for personal consumption.

Special consideration should be given to disabled athletes who may require particular transport and seating arrangements, or medications. All athletes on any medication (but particularly those such as antiepileptic drugs, insulin, or oral contraceptives) must be briefed on appropriate timing of their doses and to carry this medication in their carry on bag so that it is readily available. Also, extra contact lenses should be taken, and anaphylaxis kits if the athlete has any serious allergies. Naturally, such athletes should wear an identifying medical alert bracelet.

\section{At the venue}

All athletes are at risk of acquiring infection from unfamiliar organisms, especially those spread by the faecal-oral route or by insect vectors. This is particularly so for those who travel to developing countries. During a two week stay in such an area, between $20 \%$ and $50 \%$ of visitors develop a diarrhoeal illness. ${ }^{7}$ Most traveller's diarrhoea is due to non-viral pathogens such as enterotoxigenic Esherichia coli, Salmonella, Shigella, Campylobacter and Giardia lamblia. Many infections can be prevented by attention to food hygiene and advice to athletes includes careful hand washing before meals, ensuring that all food has been freshly cooked, and that shellfish, salads, unpasteurised milk products, and unpeeled fruits are not consumed. In many countries tap water may transmit organisms, and this should also be avoided, even in the form of ice cubes and water to brush teeth or wash food. If no bottled water is available, the local water can be boiled for at least ten minutes or water purifiers (such as iodine) can be used. Athletes should wash drinking bottles carefully as these provide ideal growing conditions for harmful organisms. For major events and when the risk of acquiring traveller's diarrhoea is relatively high, it is appropriate to use short term antibiotic prophylaxis with fluoroquinolones - for example, ciprofloxacin $500 \mathrm{mg}$ daily, doxycycline $100 \mathrm{mg}$ daily, or sulphamethoxazole with trimethoprim $800 \mathrm{mg}$ and $160 \mathrm{mg}$ daily. Note that the first two agents can produce photosensitivity.

Tropical mosquitoes can spread malaria (discussed above), dengue fever, and Japanese encephalitis. In the first six months of 1997, there was an outbreak of dengue fever in Malaysia with 7360 cases. Dengue is transmitted by mosquitoes which bite in the day time (as distinct from malaria carrying mosquitoes which bite between dusk and dawn). Protection from mosquitoes is encouraged with the use of insect repellents on the skin, sleeping in screened air conditioned rooms (and/or mosquito netting around beds), mosquito coils, and by wearing light coloured long sleeved shirts and long trousers.

The hot humid conditions of Malaysia provide excellent growing conditions for fungal infections of the skin and nails, and these are particularly likely to affect the groin and toes. Use of antibiotics increases the risk of fungal infection. Personal hygiene, keeping areas at risk clean and dry as far as possible, and frequent washing of kit greatly reduces the risk. Medical advice should be sought at the first sign of infection.

\section{HEAT AND HUMIDITY}

The heat and humidity of Malaysia will clearly be a problem for some competitors, particularly those from temperate climates who have little experience of living and competing in the heat. At the time of the Games, the daytime temperature is likely to be in the range $25-32^{\circ} \mathrm{C}$, and the humidity may range from 30 to $100 \%$. This clearly requires some preparation, and each athlete should have an individual strategy that will take care of his or her needs with regard to acclimatisation, rehydration, and lifestyle issues.

\section{ACCLIMATISATION}

It is clear that exercise performance is impaired in the heat, but that repeated exposures bring about adaptive changes that serve to minimise the negative effects on performance. The endurance trained athlete already shows some of those adaptations, particularly an expanded plasma volume, but further adaptation occurs in response to repeated bouts of exercise in the heat. There is little or no reliable information on the optimum acclimatisation programme 
for the elite athlete, but a sensible preparation would take account of the following factors.

- Acclimatisation (or acclimation) reduces the negative impact of heat on performance. It can be carried out by exercising in a hot room at home or by training in a hot climate. It is not necessary to live in the heat to become acclimatised.

- Acclimatisation is effectively achieved by exercise in the heat. Heat without exercise and exercise without heat are less effective.

- The optimum temperature for acclimatising is probably about $32-35^{\circ} \mathrm{C}$ (about 90 $95^{\circ} \mathrm{F}$ ), with a relative humidity greater than $70 \%$. About 100 minutes of exercise a day in these conditions are necessary.

- Acclimatisation takes about 7-14 days. It is not necessary for these to be consecutive days, but it is best if no more than two days elapse between exposures to prevent loss of adaptation.

- Training volume and intensity should be reduced on first exposure to the heat, and gradually increased. Some quality training should be maintained. This can be carried out in the coolest part of the day if living in a hot climate, or in the early part of the day if living at home and acclimating in a hot room.

- Some modification of the warm up before training and competition may be necessary.

\section{REHYDRATION}

Sweating allows body temperature to be maintained within reasonable limits, but results in the loss of water and electrolytes from the body. Dehydration impairs exercise performance, and probably affects mental performance as well as physical performance. The body does not adapt to dehydration, and a fluid replacement strategy that allows for adequate replacement of sweat losses is essential. As the athlete becomes acclimatised, sweat rates increase and the need for fluid intake rises accordingly.

For a typical sedentary individual in the United Kingdom, about 2-3 litres of water is lost from the body by all routes each day. In hard exercise, sweat rates are typically 1-2 litres per hour. Losses, and the need for replacement, are increased accordingly, so that the body may need perhaps 4-8 litres of water per day. When the climatic conditions are similar to those in Malaysia, the water requirement of someone taking no strenuous exercise may be about 4-6 litres per day. The increased sweat loss of the athlete in training increases the total water requirement to as much as 10 litres of fluid per day. In time, the body adjusts to cope with the need for this increased intake, and a new balance between output and intake is reestablished. It is important to note that this new balance involves an increased fluid intake with no increase in urine output; the extra fluid is all lost in sweat. For the first few days, however, the athlete, and indeed anyone going to a hot climate, will be dehydrated, until this re-adjustment has taken place. Thirst itself is not a sufficiently powerful stimulus to ensure sufficient intake of liquids in this early period of exposure, and a conscious effort must be made to drink, even when not thirsty. Athletes do not feel thirsty until a significant level of dehydration, certainly sufficient to reduce performance, is present. The same applies to support staff and team management, who should be equally aware of the dangers of dehydration. This does not mean that large amounts should be consumed constantly, as water intoxication may result. Body weight should be used as a guide.

Most people will arrive in Malaysia already dehydrated as a result of fluid losses during the flight. This is a result of the low water vapour pressure in the cabin which increases fluid loss from the body. The flight time from London to Malaysia is long enough to lose a large amount of fluid in this way. Very few travellers drink enough while flying to compensate for these losses and extra fluids during the flight should be encouraged.

The optimum fluid for rehydration depends on the sport, on the individual, and on a number of other factors. Drinking large volumes of plain water depresses the serum sodium concentration and lowers plasma osmolality, both of which have the effect of inhibiting the sensation of thirst (thus reducing intake) and of stimulating urine output (thus increasing water loss from the body). Addition of electrolytes, of which sodium is the only one of significance, helps to prevent this. Addition of some sugar stimulates intestinal water absorption and acts as an energy substrate, supplementing the body's limited carbohydrate stores. When the need for water replacement is high because of long periods spent outdoors but the rate of energy expenditure is relatively low, as may be the case in shooting or bowls for example, the carbohydrate content of drinks should be kept low and the sodium content relatively high. Where the energy demand is high, a higher carbohydrate content may be beneficial.

If dehydration is used to make weight in weight category sports (such as boxing), every effort should be made to rehydrate as completely as possible after the weigh in. Again, dilute carbohydrate-electrolyte drinks are the most effective way of doing this.

It is worth remembering that dehydration abolishes the benefits of acclimatisation. The fully acclimatised individual who becomes dehydrated responds to heat stress as if completely unacclimatised. Another potential problem is traveller's diarrhoea, which also increases fluid losses. This may be impossible to avoid and it is highly probable that some team members will suffer at some stage. Treatment includes an increase in the intake of fluids and one may consider antibiotics in more severe or persisting cases. Sports drinks or oral rehydration fluids specifically designed for the treatment of diarrhoea should be taken. Water, fruit juices, and cola drinks are less effective, either because they contain no electrolytes or have too little or too much sugar. An appropriate antibiotic treatment is ciprofloxacin (250 mg twice a day) or trimethoprim (200 mg twice a day) for five days. The feeling of tiredness that comes with this type of diarrhoea is largely the 
result of dehydration (as is the muscle stiffness and increased delayed onset muscle soreness) and can be overcome with suitable fluid replacement.

Finally, one should not forget the support staff. They too are at risk of dehydration and heat illness, and should take appropriate steps along the lines indicated above to prevent this. Team officials are often required to spend long periods exposed to the sun, and must ensure both protection from solar radiation and appropriate fluid intake. Mental performance and physical performance are impaired by dehydration and hyperthermia.

Key points:

- Extra fluids should be consumed during the flight to prevent arrival in a dehydrated state.

- Fluid loss is greatly increased in the heat. Daily fluid requirements may increase by 2-3 times normal.

- Dehydration impairs performance and the body does not adapt to dehydration.

- An adequate intake of an appropriate fluid before, during (where appropriate), and after training and competition is essential.

- Individuals must experiment in training to establish the most effective fluid replacement strategy. Daily (morning) body weights may be useful and each athlete's urine should be clear, not dark.

\section{LIFESTYLE ISSUES}

People unaccustomed to living in the heat take time to adapt and to learn coping strategies. Avoiding the sun does not come easily to those who seldom see it, but sunburn can be a major problem. Sunburn, even to a minor degree, increases the heat strain during training by reducing the sweating response. This effect can persist for at least two weeks after only a minor degree of skin damage, long after the subjective symptoms have disappeared. Solar radiation should therefore be avoided by avoiding direct exposure where possible and by use of suitable clothing and sunscreens. A hat and a T-shirt (not sleeveless vest) are sensible. Sunscreens to be used should block UVA and UVB light. A high protection factor (greater than 15) should be used in all fair skinned individuals. Only those who know that they tan quickly and easily should use anything less. Sunscreens should be reapplied frequently, especially when the skin is wet, and this means almost all the time. Even when the sky is overcast, which it will be most of the time, the risk of burning is high.

Loose fitting cotton clothing such as cotton mesh is most effective in helping evaporation of sweat and heat dissipation. Underclothes should be made of a material that is comfortable even when wet or one that wicks the water away from the skin. These should be tried in training at home. White or light coloured clothing is effective at reflecting heat and prevents it from being absorbed by the body. Black or dark clothing maximises the absorption of solar radiation. Wearing a hat can greatly reduce the solar radiation gained through the head, and the difference between wearing a black hat and a white one can be substantial. Opportunities to make use of available shade should be taken wherever possible.

Alcohol has many negative effects, including a tendency to cause an increased urine output, and this becomes a significant factor when drinks with a high alcohol content (spirits, wine etc) are consumed. The caffeine contained in tea and coffee has the same effect. These drinks are consumed in small volumes (usually!), and the urine output stimulated by the effect of alcohol is greater than the volume of fluid consumed and may contribute to dehydration. Drinks with a low alcohol content (weak beer, shandy, wine cooler) may be acceptable for some, but should not form a large part of the day's total fluid intake. Tea and coffee should not be too strong, but need not be discontinued by the athlete who is used to drinking them.

Key points:

- Minimise exposure to the sun. Wear appropriate clothes and use sunblock.

- Avoid excess alcohol and large amounts of caffeine.

\section{Conclusion}

For many athletes, the 1998 Commonwealth games will be the highlight of their sporting career. For all competitors it will be a major milestone, and for medal winners it will be the achievement of a lifetime. The role of the medical team is to ensure that all their athletes arrive at the venue in good health, remain in good health, and are protected against any potential serious illness. The key to a successful outcome is preparation and teamwork, and the sign of success will be a quiet medical room at the games. The best medical team is one that is not seen, not because they are unavailable, but because they are not needed.

The authors wish to thank Dr Bernie Hudson and Chris Birch of the Medical Advisory Service for Travellers Abroad, Sydney, New South Wales, for their helpful advice. Dr Elizabeth McSwan, of the Scottish Commonwealth Games team gave advice on immunisations. Professor Jack Taunton and Professor Tim Noakes gave very helpful advice and criticism, and suggested additions to the text. The paper was peer reviewed by Jack Taunton and Tim Noakes. It was an open review with authors and reviewers knowing each others' identities. The idea for this paper was first raised at an editorial board meeting in Denver in 1996 with a view to the forthcoming Commonwealth Games. The subject, authorship, and review spans five continents. The paper was written in Australia (MY, PF) and the United Kingdom (RM, written in Australia ( $\mathrm{MY}, \mathrm{PF}$ ) and the United Kingdom (RM, DMacA) and reviewed in Canada (JT) and South Africa (TN). The process was completed almost
entirely using electronic media.

1 Bock HL, Sanger R, Ahrens K, et al. Interscience conference on antimicrobial agents and chemotherapy (ICAAC). Orlando,
Florida, October 1994.

2 Barrett PJ, Emmins PD, Clarke PD, Bradley DJ. Comparison of adverse events associated with use of mefloquine and combination of chloroquine and proguanil as antimalarial prophylaxis: postal and telephone survey of travellers. BMF 996;313:525-8.

3 Shephard RJ. Sleep biorhythms and human performance. Sports Med 1984;1:11-37.

4 O'Connor PJ, Morgan WP. Athletic performance following rapid traversal of multiple time zones. Sports Med 1990;10: 20-30.

5 Petrie K, Dawson AG, Thompson L, Brook R. A double-blind trial of melatonin as a treatment for jet lag in international cabin crew. Biol Psychiatry 1993;33:526-30.

6 Petrie K, Conaglen JV, Thompson L, Chamberlain K. Effect of melatonin on jet lag after long haul flights. BMF 1989;298:705-7.

7 Korman TM, Ruff TA. Traveller's diarrhoea. Modern Medicine of Australia 1997;40:66-74.

8 Maughan RJ. Fluid and electrolyte loss and replacement in exercise. In: Harries M, Williams C, Stanish WD, Micheli LJ, eds. Oxford textbook of sports medicine Oxford: Oxford University Press, 1994:82-94. 